EVALUATION OF QUOTA HUNTING FOR COUGAR IN ALBERTA DURING 1990-1997

By

John R. Gunson

Occasional Paper Number 18 Wildlife Management Division

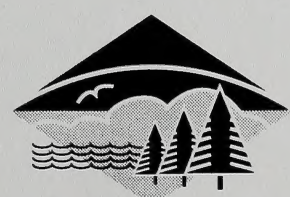

Alberia 
Digitized by the Internet Archive in 2016 


\section{EVALUATION OF QUOTA HUNTING FOR COUGAR IN ALBERTA DURING 1990-1997}

By

John R. Gunson

Alberta

Environmental Protection

Edmonton

Natural Resources Service

Wildlife Management Division 
Pub. No.: $T / 388$

ISBN: 0-7732-9995-5

For copies of this document, please contact:

Information Centre

Alberta Environmental Protection

Main Floor, Bramalea Building

9920 - 108 Street

Edmonton, Alberta, Canada T5K 2M4

Telephone: (403) 422-2079 


\section{CONTENTS}

\begin{tabular}{|c|c|}
\hline & - \\
\hline & RESULTS................... \\
\hline 2.1 & Quotas.. \\
\hline 2.2 & Harvest............................. \\
\hline 2.3 & Timing of the Harvest....... \\
\hline 2.4 & Closures.. \\
\hline 2.5 & Hunter Effort, Participation and Success......................... \\
\hline 2.6 & Cougar Sex Ratio............. \\
\hline 2.7 & Age Structure \\
\hline 2.8 & Total Mortalities........................... \\
\hline & DISCUSSION.. \\
\hline & LITERATURE CITED.. \\
\hline
\end{tabular}




\section{LIST OF FIGURES}

Figure

Page

1. Cougar Management Areas in Alberta.................................................. 3 


\section{LIST OF TABLES}

Table

1. Comparison of total cougar hunting quotas and harvests in Alberta....... 4

2. Comparison of female cougar hunting quotas and harvests in Alberta... 6

3. Harvests of cougar in Alberta: by sex................................................ 7

4. Weekly harvest of cougar by Cougar Management Area during 1990-91 to $1996-97$.

5. Summary of closures in Cougar Management Areas.

6. Success in resident cougar hunting by Cougar Management Area during 5 years $(-91,-93,-94,-95,-96)$.

7. Success in cougar hunting in Alberta.

8. Sex ratios of cougar harvested in Alberta

9. Sex ratios of cougar harvested in Alberta by week.

10. Summary of skull classes of harvested cougar in Alberta.

11. Summary of total man-caused mortalities for cougar in Alberta. 


\section{ACKNOWLEDGMENTS}

Alberta Natural Resources Service district personnel are acknowledged for completion of registration certificates. Sylvia Birkholz compiled registrations. Bruce Treichel coordinated skull collections and classification. Brent Markham reviewed an earlier draft of this report. 


\subsection{INTRODUCTION}

Cougar have been hunted in Alberta throughout the period of European settlement to the present; however, prior to 1965 , the species was managed as a bountied predator. Between 1937 and 1964, an annual average of 40 cougar were taken for bounty. Beginning in the 1960s, cougar were harvested under the authority of a deer, elk or moose recreational hunting licence. In 1971, cougar were classified as "big game" and a cougar hunting licence was introduced, although a cougar could still be harvested by the holder of an elk hunting licence until 1972. By 1973, cougar could only be harvested by the holder of a valid cougar hunting licence.

Cougar were hunted without dogs in a 80-100-day fall season (that corresponded to the seasons for other big game species); with dogs during a 50-60-day winter season (December-January, 1971-1977), during January, 1978-1990 (Wildlife Management Units [WMUs] 300-312) and during the 2-month season in the remainder of WMUs, and only during January, 1981-1990 (all WMUs). The fall season was shortened to 66 days in 1979 and was entirely eliminated in 1985 . Hunters with a valid cougar licence (no restriction to the number of licences sold) could hunt in any WMU with a cougar season to the end of the season that closed at the same time in all areas. Because of the short winter season and unpredictable weather, harvests fluctuated widely from one year to another; mean annual harvest during 1978-1990 (12 years) was 33 (range 21-47).

This management of cougar as a game animal was complicated by several factors (Ross et al. 1996). First, most cougar houndsmen in Alberta lived and hunted in the south with much less hunting and lower harvests in more-northern areas. Second, reliable inventory over the large range of this reclusive species in Alberta is not possible. Third, because of the relative ease of hound-assisted hunting, individual cougar are vulnerable to harvest and local overharvests can occur. Fourth, cougar sex can be difficult to determine; some unintentional harvest of females can be expected. Last, all-season breeding and birth of young by cougar in Alberta means that some orphaning of young probably results from hunting harvests. 
Beginning in the 1980s, cougar houndsmen reported abundant cougar and requested more liberal seasons. Cougar populations and hunting management were reviewed and the resulting analyses were published in a management plan (Alberta Fish and Wildlife Division 1992). The plan recommended a new hunting system for cougar and this was initiated in the 1990-91 season. A regional harvest quota became the basis for the new system. The huntable cougar range in Alberta was divided into 11 regional Cougar Management Areas (CMAs; Figure 1). Based on habitat-specific estimates of prey and cougar populations with intensive data from the Sheep River study (Ross and Jalkotzy 1992), CMA cougar populations were estimated and quotas were established. For each CMA, there was both a total-harvest quota and a female-harvest quota. Harvest quotas were replaced by total man-caused mortality quotas beginning in 1995-96. Successful cougar hunters were required to register their kill within two business days and (beginning in 1992) to provide the carcass for confirmation of sex. When either the total or female quota were reached, the season in that CMA closed (this was announced on a 1-800 telephone line and by registered letter). As a rule, hunters were given a grace period of several days to terminate hunting in that CMA.

This report summarizes cougar hunting during 1990-1997 and evaluates the effectiveness of the evolving new hunting system.

\subsection{RESULTS}

\subsection{Quotas}

Total hunting quotas during 1990-91 to $1994-95$ were set at 10 percent of the estimated CMA cougar population as established in the provincial cougar management plan (quotas in 1990-91 [total of 58] were based on incomplete population data). Provincial total quota was 66 with a range of 2-9 in individual CMAs (Table 1). During 1995-1997 (last 2 years), the 10 percent total hunting quota was replaced by a 15 -percent man-caused mortality quota and all man-caused mortalities were considered; provincial total quota was 89 (range 2-14). Female quotas were approximately one-half of total quotas during all 


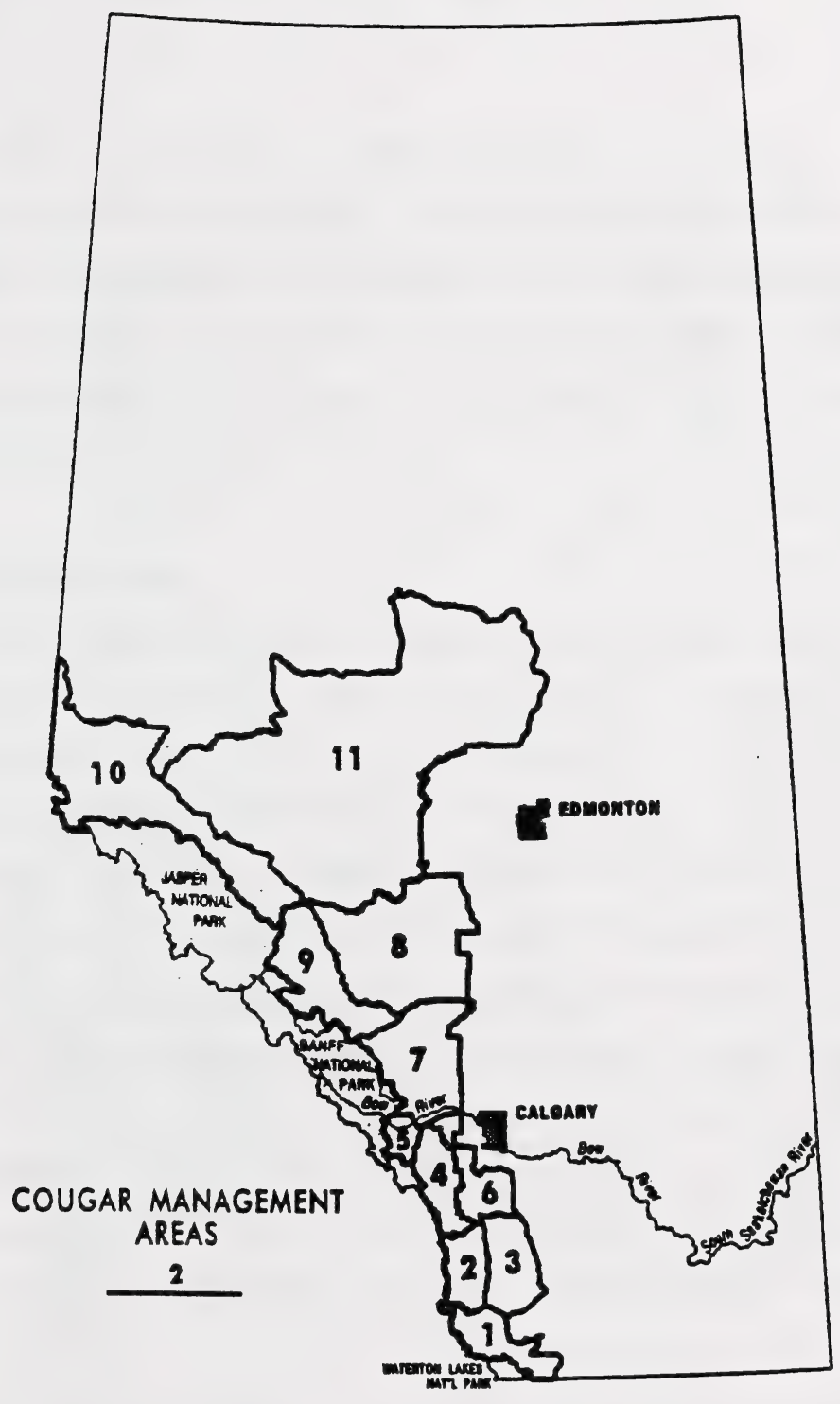

Figure 1. Cougar Management Areas in Alberta 


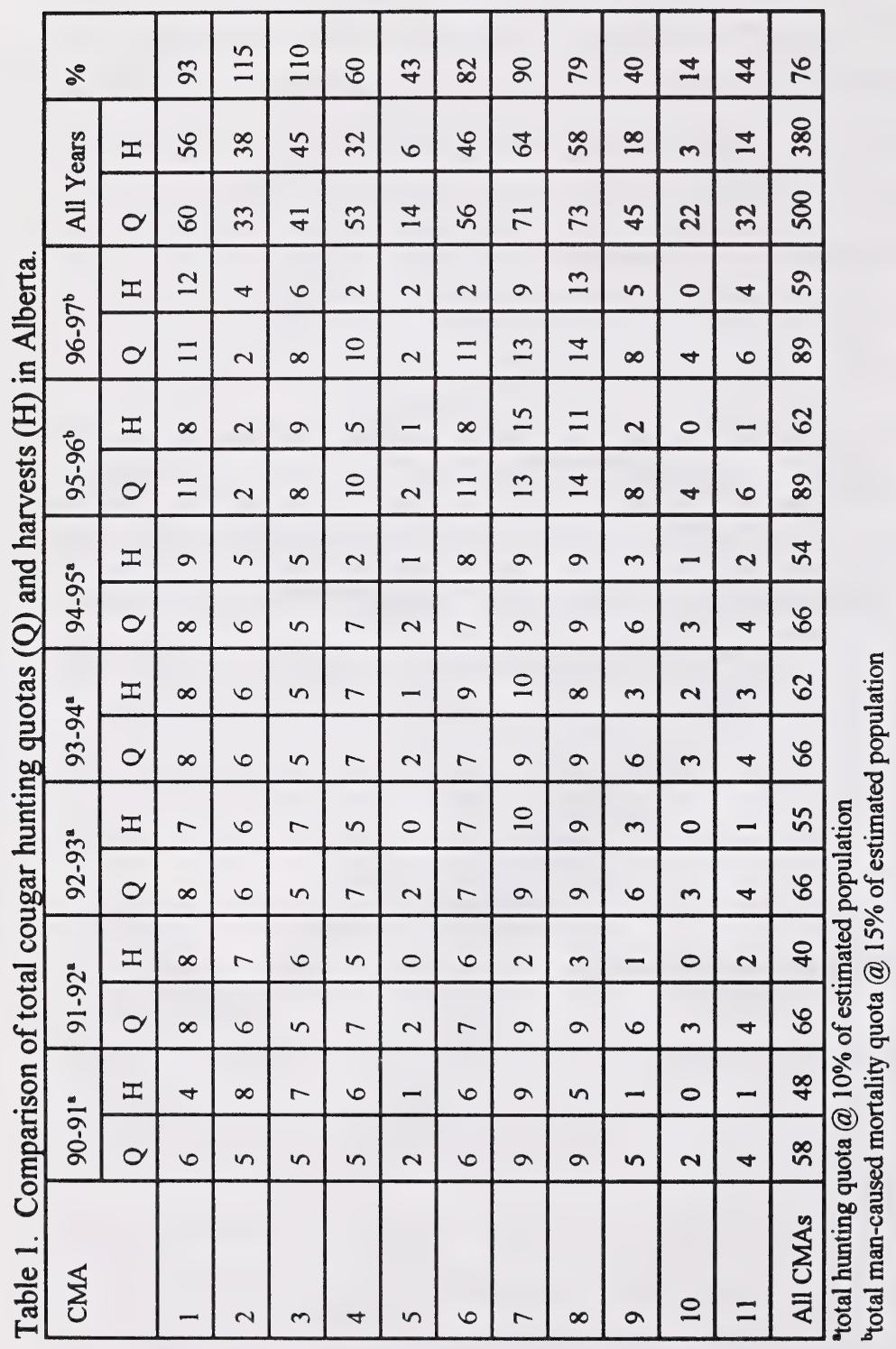


years (Table 2; 1991-95=total of 36, 1995-97=total of 45).

\subsection{Harvest}

Three hundred and eighty cougars were taken by licenced hunters during the 7 seasons of which 268 ( 71 percent) were males (Table 3). Annual provincial harvests ranged between 40 (1991-92) to 62 (1993-94 and 1995-96). Multi-year harvests in individual CMAs were 14 percent (CMA 10) to 115 percent (CMA 2) of total quotas (Table 1) and 8 percent (CMA 10) to 71 percent (CMA 2) of female quotas (Table 2). Over all CMAs, 76 percent of the entire total quota of 500 was harvested.

\subsection{Timing of the Harvest}

Weekly harvests were generally, but not always, greatest during week 1 and week 2 of the season (first 2 weeks of December; over the 7 years, 31 percent of the harvest occurred during these 2 weeks; Table 4). Harvest usually peaked again in January (weeks 5 to 8 of the season), but this peak was usually somewhat less than that during the beginning of the season. Harvests during the latter 2 weeks of December were undoubtedly influenced by the Christmas season. On a monthly basis, 186 cougar ( 49 percent) were taken in December, 163 (43 percent) in January and 31 (8 percent) in February.

Annual variation in timing of the harvest was importantly influenced by snow and tracking conditions. For example, very cold weather (daily minimum temperatures of $-30^{\circ}$ to $-35^{\circ} \mathrm{C}$ ) during mid December, 1990 prevented the use of hounds and almost entirely eliminated cougar harvest for 2 weeks. During the following year, harvest in the south slowed during late January and early February because of lack of snow.

\subsection{Closures}

Number of annual CMA closures (based on attainment of the total or female quotas) varied from 3 to 7 and averaged 5 (Table 5). Of 37 closures, 32 ( 86 percent) resulted from the total quota being reached and 5 (14 percent) from the female subquota. Eight (22 percent) of the closures occurred in December, 23 (62 percent) in January and 6 (16 
Table 2. Comparison of female cougar hunting quotas $(\mathrm{Q})$ and harvests $(\mathrm{H})$ in Alberta.

\begin{tabular}{|c|c|c|c|c|c|c|c|c|c|c|c|c|c|c|c|c|c|}
\hline \multirow[t]{2}{*}{ CMA } & \multicolumn{2}{|c|}{$90-91^{2}$} & \multicolumn{2}{|c|}{$91-92^{2}$} & \multicolumn{2}{|c|}{ 92-93" } & \multicolumn{2}{|c|}{$93-94^{\circ}$} & \multicolumn{2}{|c|}{$94-95^{a}$} & \multicolumn{2}{|c|}{$95-96^{b}$} & \multicolumn{2}{|c|}{$96-97^{b}$} & \multicolumn{2}{|c|}{ All Years } & \multirow[t]{2}{*}{$\%$} \\
\hline & Q & $\mathrm{H}$ & $\mathrm{Q}$ & $\mathrm{H}$ & Q & $\mathrm{H}$ & $\mathrm{Q}$ & $\mathrm{H}$ & $\mathrm{Q}$ & $\mathrm{H}$ & $\mathrm{Q}$ & $\mathrm{H}$ & $\mathrm{Q}$ & $\mathrm{H}$ & Q & $\mathrm{H}$ & \\
\hline 1 & 3 & 1 & 4 & 1 & 4 & 1 & 4 & 4 & 4 & 2 & 6 & 4 & 6 & 5 & 31 & 18 & 58 \\
\hline 2 & 3 & 5 & 3 & 1 & 3 & 2 & 3 & 2 & 3 & 1 & 1 & 0 & 1 & 1 & 17 & 12 & 71 \\
\hline 3 & 3 & 2 & 3 & 3 & 3 & 1 & 3 & 1 & 3 & 1 & 4 & 2 & 4 & 3 & 23 & 13 & 57 \\
\hline 4 & 3 & 2 & 4 & 1 & 4 & 1 & 4 & 2 & 4 & 0 & 5 & 3 & 5 & 1 & 29 & 10 & 34 \\
\hline 5 & 1 & 0 & 1 & 0 & 1 & 0 & 1 & 0 & 1 & 1 & 1 & 1 & 1 & 1 & 7 & 3 & 43 \\
\hline 6 & 3 & 2 & 4 & 1 & 4 & 1 & 4 & 3 & 4 & 1 & 5 & 2 & 5 & 0 & 29 & 10 & 34 \\
\hline 7 & 5 & 5 & 5 & 0 & 5 & 0 & 5 & 4 & 5 & 3 & 7 & 2 & 7 & 4 & 39 & 18 & 46 \\
\hline 8 & 5 & 2 & 5 & 1 & 5 & 3 & 5 & 4 & 5 & 2 & 7 & 4 & 7 & 2 & 39 & 18 & 46 \\
\hline 9 & 3 & 0 & 3 & 1 & 3 & 0 & 3 & 0 & 3 & 1 & 4 & 1 & 4 & 0 & 23 & 3 & 13 \\
\hline 10 & 1 & 0 & 2 & 0 & 2 & 0 & 2 & 0 & 2 & 1 & 2 & 0 & 2 & 0 & 13 & 1 & 8 \\
\hline 11 & 2 & 0 & 2 & 0 & 2 & 1 & 2 & 1 & 2 & 2 & 3 & 0 & 3 & 1 & 16 & 5 & 31 \\
\hline All CMAs & 32 & 19 & 36 & 9 & 36 & 10 & 36 & 21 & 36 & 16 & 45 & 19 & 45 & 18 & 227 & 112 & 49 \\
\hline
\end{tabular}

' female hunting quota @ one-half of total hunting quota

b female quota @ one-half of total man-caused mortality quota 


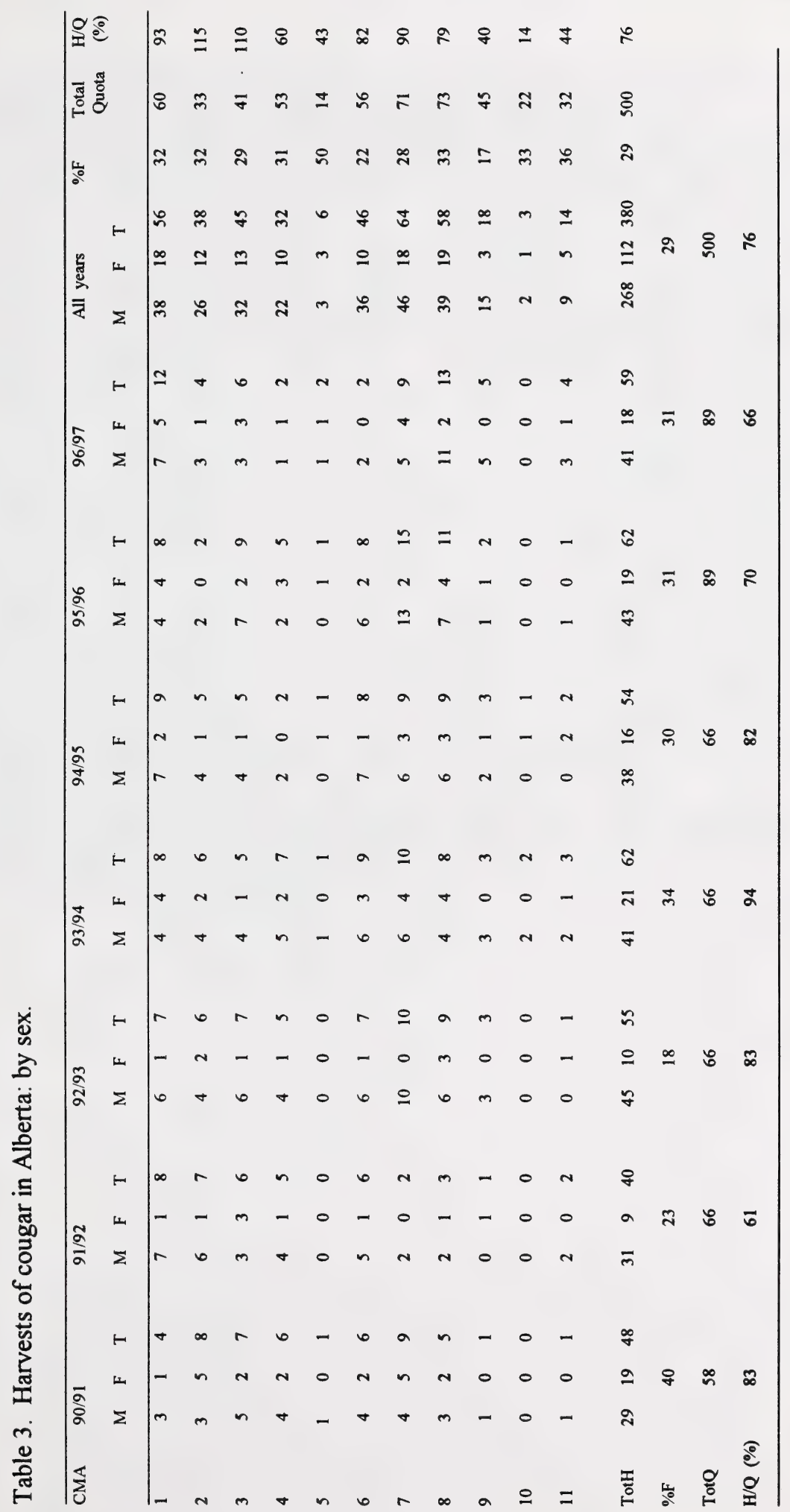


Table 4. Weekly harvest of cougar by Cougar Management Area during 1990-91 to 1996-97.

\begin{tabular}{|c|c|c|c|c|c|c|c|c|c|c|c|c|c|c|}
\hline \multirow{2}{*}{ CMA } & \multicolumn{4}{|c|}{ December } & \multicolumn{4}{|c|}{ January } & \multicolumn{5}{|c|}{ February } & \multirow{2}{*}{ Total } \\
\hline & 7 & 14 & 21 & 28 & 4 & 11 & 18 & 25 & 1 & 8 & 15 & 22 & 28 & \\
\hline 01 & 5 & 10 & 5 & 2 & 8 & 7 & 10 & 4 & 2 & 1 & & 2 & & 56 \\
\hline 02 & 3 & 5 & 2 & 5 & 4 & 8 & 5 & 6 & & & & & & 38 \\
\hline 03 & 20 & 13 & 4 & 1 & 2 & 1 & 1 & 1 & 2 & & & & & 45 \\
\hline 04 & 3 & 8 & 2 & 1 & 2 & 3 & 5 & 2 & 3 & & 2 & 1 & & 32 \\
\hline 05 & 1 & & & & 1 & & 1 & & 2 & & & & 1 & 6 \\
\hline 06 & 5 & 4 & 2 & 3 & 5 & 8 & 5 & 3 & 5 & 2 & 2 & 1 & 2 & 47 \\
\hline 07 & 8 & 9 & 7 & 2 & 12 & 8 & 7 & 2 & 3 & & 1 & 1 & 3 & 63 \\
\hline 08 & 10 & 6 & 3 & 4 & 9 & 8 & 9 & 3 & 1 & & 2 & 1 & 2 & 58 \\
\hline 09 & 2 & 1 & 1 & 2 & 2 & 1 & 3 & 1 & & 1 & 1 & 1 & 2 & 18 \\
\hline 10 & & 1 & 1 & & & 1 & & & & & & & & 3 \\
\hline 11 & 3 & 2 & 1 & 1 & 2 & 1 & 2 & 1 & 1 & & & & & 14 \\
\hline $\begin{array}{l}\text { All } \\
\text { CMAs }\end{array}$ & 60 & 59 & 28 & 21 & 47 & 46 & 48 & 23 & 19 & 4 & 8 & 7 & 10 & 380 \\
\hline
\end{tabular}


Table 5. Summary of closures in Cougar Management Areas.

\begin{tabular}{|c|c|c|c|c|c|c|c|}
\hline CMA & $1990-91$ & 1991-92 & $1992-93$ & $1993-94$ & $1994-95$ & $1995-96$ & $1996-97$ \\
\hline 1 & & Feb3 & & $\operatorname{Jan} 20$ & Jan 16 & & Feb1 \\
\hline 2 & Jan19 & Jan9 & Jan14 & Jan17 & & Dec2* & Jan $4 *$ \\
\hline 3 & Dec22 & Dec11-F & Dec17 & $\operatorname{Jan} 7$ & Dec9 & Dec13 & Dec5-F \\
\hline 4 & $\operatorname{Jan} 29$ & & & $\operatorname{Jan} 20$ & & & \\
\hline 5 & & & & & Jan2-F & Dec5-F & Feb28 \\
\hline 6 & Jan 29 & & Jan11 & $\operatorname{Jan} 8$ & Feb9 & Feb10 & \\
\hline 7 & $\operatorname{Jan} 25$ & & Feb23 & $\operatorname{Jan} 15$ & $\operatorname{Jan} 18$ & Jan6 & \\
\hline 8 & & & Jan4 & & $\operatorname{Jan} 20$ & & Jan15 \\
\hline \multicolumn{8}{|l|}{9} \\
\hline \multicolumn{8}{|l|}{10} \\
\hline 11 & & & & & $\operatorname{Jan} 26-\mathrm{F}$ & & $\operatorname{Jan} 28$ \\
\hline $\begin{array}{l}\text { Number of } \\
\text { Closures }\end{array}$ & 5 & 3 & 5 & 6 & 7 & 5 & 6 \\
\hline
\end{tabular}

* quotas were lower $(\mathrm{F}=1, \mathrm{~T}=2)$ than in previous years

-F closure due to attainment of female subquota 
percent) in February. Management areas most commonly closed were CMA 3 (all 7 years), CMA 2 (6 of 7 years) and CMAs 6 and 7 (5 years each).

Harvest (or total mortalities [1995-96,1996-97]) exceeded total quotas in some CMAs every year. There were 17 such cases with a total of 32 cougar of which 12 (38 percent) were females. On a multi-year basis, total quotas were exceeded only in CMA 2 ( 5 cougar) and CMA 3 (4 cougar).

\subsection{Hunter Effort, Participation and Success}

Information on hunting effort and success is provided by hunters in annual telephone surveys coordinated by government and carried out by volunteer organizations like the Alberta Fish and Game Association. With cougar, resulting data are available for 5 years (1990-91, 1992-93, 1993-94, 1994-95, 1995-96).

Since 1973, when the cougar licence was introduced, between 54 (1973) and 173 (1979) cougar hunting licences have been sold annually. Since 1990, when the new hunting system began, annual licence sales for residents ranged from 113 to 146, and for non-residents, 5 to 20 . However, not all licencees are active in any year; this is especially the case with resident hunters. According to results of hunter telephone surveys for the 5 years, 80 percent (659 licencees, 530 hunters) of residents hunted. On annual average then, 106 residents and 12 non-residents hunted cougar.

Data relative to the number of days hunted show a range from 3 days ( 42 days hunting by 14 hunters) in CMA 5 ( a high-country unit with more-difficult access), 4 days (385 days, 96 hunters) in CMA 3 (best access, good cougar population) to 14 days (58 days, 4 hunters) in CMA 10 (a remote, northern unit with a dispersed cougar population). Overall, hunters spent an average of 10 days hunting cougar with a mean of 1.7 CMAs used per hunter per year.

In those CMAs $(1,2,3,6,7,8)$ where hunting is most intensive (greater than 90 hunters during 5 years), CMA-specific success rates of residents varied between 21 and 38 percent (Table 6). Because most resident hunters pursue cougar in more than one CMA, average annual success was 36 percent (range 30 percent in 1991-92 to 43 percent in 
Table 6. Success ${ }^{2}$ in resident cougar hunting by Cougar Management Area in Alberta during 5 years $(-91,-93,-94,-95,-96)$.

\begin{tabular}{|l|c|c|c|}
\hline CMA & $\begin{array}{c}\text { Estimated number } \\
\text { of hunters } \\
\text { (total of } 5 \text { yrs.) }\end{array}$ & $\begin{array}{c}\text { Harvest } \\
(5 \text { yrs.) }\end{array}$ & Success (\%) \\
\hline 1 & 163 & 34 & 21 \\
\hline 2 & 119 & 25 & 21 \\
\hline 3 & 96 & 31 & 32 \\
\hline 4 & 73 & 20 & 27 \\
\hline 5 & 14 & 4 & 29 \\
\hline 6 & 96 & 34 & 35 \\
\hline 7 & 123 & 47 & 38 \\
\hline 8 & 130 & 35 & 27 \\
\hline 9 & 21 & 7 & 33 \\
\hline 10 & 4 & 2 & 50 \\
\hline 11 & 42 & 8 & 19 \\
\hline
\end{tabular}

a average annual success for all resident cougar hunters was $36 \%$ (see Table 7); because hunters use more than one CMA, (average of $1.7 \mathrm{CMAs}$ /hunter), success in any individual area is generally less. ${ }^{b}$ from annual telephone surveys of cougar hunters and adjusted for participation (annual average of 80 percent). 
1992-93; Table 7). Non-resident hunters are guided and their hunts averaged 60 percent success (annual range:33-80 percent).

\subsection{Cougar Sex Ratio}

Hunters in Alberta prefer to shoot the largest cougar, which are males. Once treed, cougar sex can be determined reasonably well. Annual sex ratios in the total provincial harvest ranged between 60 and 82 percent males (Table 8). On a CMA basis, sex ratios below 2/3 males occured only in CMAs 5 (low harvest, 50 percent males) and CMA 11 (64 percent males). In the heaviest hunted areas (CMAs 1,2,3,6,7,8), male proportion in the harvest varied only between 67 and 78 percent.

Given a preference by hunters for large, male cougar, one might have expected selection for males early in the season followed by a gradual increase in female cougar as the seasons approach closure. This scenario would be expected if cougar were scarce. When CMAs are combined (that is, total provincial harvest, Table 9), there is no such relationship of increasing female harvests as the season progresses which may be suggestive of a healthy cougar population. As a rule then, closures may have been successful in adequately protecting females and maintaining populations. However, cougar hunting guides (B. Sinclair, G. Crouch, pers. comun., 1997) have noted regional scarcity of large males in recent years. CMA-specific data seem to support this (e.g., CMA 3--50 percent males in 1996-97 following several years of high male harvests; CMA 7--56 percent males in 1996-97 following strong harvest of males the preceding year.

\subsection{Age Structure}

Hunters have voluntarily (to 1988) and, as required since 1989, submitted skulls from harvested cougar to Alberta Fish and Wildlife for classification to age. Greer's (1972) system of classifying skulls was employed. Individual and annual average classifications from 283 cougar taken by hunters during 7 years are in Table 10. There is no suggestion of declining cougar age in the harvest in any CMA. Fifteen subadults (independent kitten [class I], yearlings [class II) represented 5 percent of the sample; 122 young adults (classes 


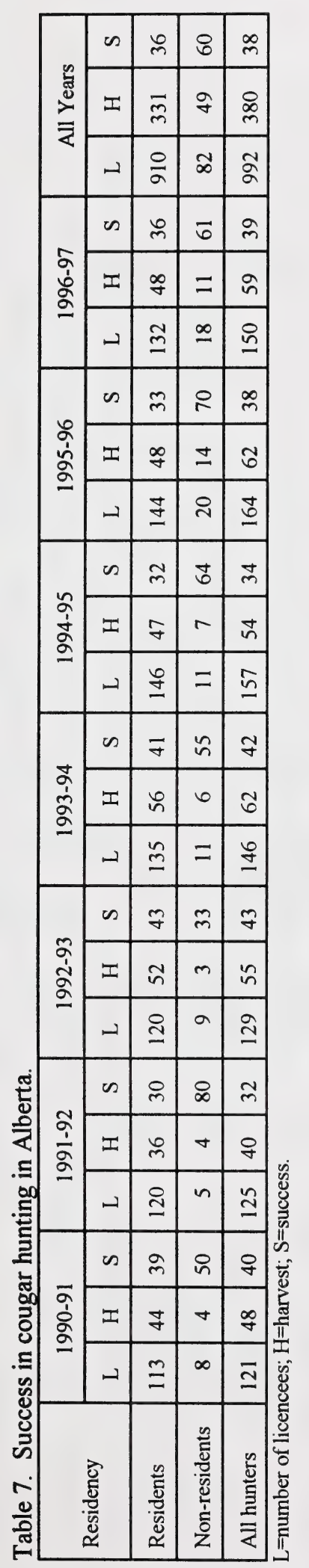




\begin{tabular}{|c|c|c|c|c|c|c|c|c|c|c|c|c|c|c|c|}
\hline & $\underline{\alpha}$ & & & $\approx$ & 8 & 5 & & 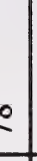 & $\approx$ & 5 & $\infty$ & 5 & t & $\approx$ & \\
\hline$\equiv$ & $\frac{\alpha}{\dot{\alpha}}$ & & & $\stackrel{m}{i}$ & $\stackrel{0}{\ddot{\sim}}$ & 3 & & $\stackrel{0}{0}$ & $\begin{array}{l}\stackrel{\infty}{\ddot{\dot{b}}} \\
\dot{+}\end{array}$ & $\frac{a}{\dot{m}}$ & $\stackrel{m}{\tilde{n}}$ & $\overline{\dot{\sim}}$ & $\tilde{a}$ & 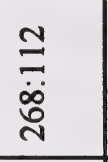 & $\approx$ \\
\hline 5 & r & & & $\stackrel{m}{m}$ & $\Xi$ & 3 & & i. & $\ddot{n}$ & $\stackrel{\stackrel{N}{=}}{=}$ & i. & $\dot{0}$ & $\bar{m}$ & $\stackrel{\infty}{\stackrel{\vec{\sigma}}{\vec{\sigma}}}$ & 8 \\
\hline 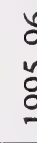 & $\dot{y}$ & & & $\stackrel{\Upsilon}{\sim}$ & $\stackrel{m}{\sim}$ & 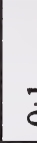 & & $\begin{array}{c}y \\
0.1 \\
0\end{array}$ & 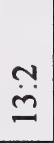 & 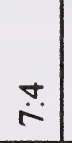 & $\ddot{ت}$ & 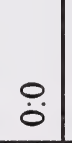 & $\stackrel{\circ}{-}$ & $\frac{a}{\ddot{q}}$ & 8 \\
\hline 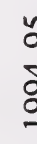 & i & & & $\overrightarrow{\dot{\sigma}}$ & $\stackrel{\text { i }}{ }$ & 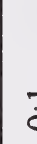 & & 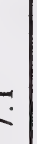 & ్ֶర & $\tilde{b}$ & $\vec{\sim}$ & $\ddot{0}$ & $\stackrel{\Upsilon}{0}$ & $\underset{\infty}{\infty}$ & $\approx$ \\
\hline 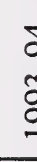 & $\dot{\vec{v}}$ & & & $\ddot{\forall}$ & $\tilde{n}$ & 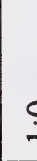 & & है. & $\ddot{\dot{\theta}}$ & $\begin{array}{l}\forall \\
\dot{\forall}\end{array}$ & $\stackrel{\dot{m}}{.}$ & $\stackrel{\circ}{i}$ & $\bar{i}$ & $\stackrel{\bar{ָ}}{\vec{\forall}}$ & \\
\hline $\begin{array}{l}\tilde{\delta} \\
\delta \\
\delta\end{array}$ & $\bar{b}$ & & & $\overline{0}$ & $\overline{\dot{\gamma}}$ & 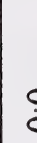 & & i. & $\dot{0}$ & $\hat{0}$ & $\stackrel{i}{i}$ & $\ddot{0}$ & $\overline{0}$ & $\overrightarrow{\ddot{g}}$ & $\approx$ \\
\hline$\delta$ & $\bar{i}$ & & & $\stackrel{m}{m}$ & $\overline{\dot{y}}$ & 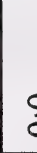 & & 8 & $\stackrel{\circ}{i}$ & $\bar{i}$ & $\overrightarrow{0}$ & $\stackrel{0}{0}$ & $\stackrel{\circ}{i}$ & $\stackrel{o}{m}$ & $\stackrel{\infty}{\Upsilon}$ \\
\hline & $\bar{i}$ & & & $\ddot{n}$ & $\dot{F}$ & 9 & & j & $\stackrel{n}{\dot{\sigma}}$ & $\stackrel{\sim}{i}$ & $\stackrel{\circ}{-}$ & $\dot{0}$ & $\stackrel{\circ}{-}$ & $\frac{a}{a}$ & 8 \\
\hline & - & & & $m$ & 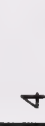 & & & 0 & - & $\infty$ & $a$ & 이 & $=$ & ₹ & 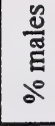 \\
\hline
\end{tabular}




\begin{tabular}{|c|c|c|c|c|c|c|c|c|c|c|c|c|c|c|c|}
\hline \multicolumn{2}{|c|}{$0 \frac{\mathscr{U}}{\Xi}$} & $\approx$ & $\bar{N}$ & $\tilde{\infty}$ & $\stackrel{2}{2}$ & $\mathbb{N}$ & $\bar{\infty}$ & $\widehat{6}$ & $\stackrel{\infty}{+}$ & $\hat{6}$ & 으 & $\stackrel{8}{ }$ & $\hat{\sigma}$ & $\hat{b}$ & $\pi$ \\
\hline \multirow{2}{*}{ 离 } & LI & $\bar{\sim}$ & $I$ & $n$ & $n$ & $\underline{m}$ & $a$ & 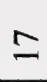 & $m$ & 6 & 0 & 0 & $m$ & $m$ & $\cong$ \\
\hline & $\Sigma$ & m & $\bar{\nabla}$ & $\stackrel{\sim}{\sim}$ & $\underline{0}$ & $\stackrel{ \pm}{m}$ & $\stackrel{\infty}{m}$ & নิ & $\simeq$ & $\simeq$ & $m$ & $a$ & 0 & $n$ & $\stackrel{\infty}{\infty}$ \\
\hline \multirow{2}{*}{ ấ } & [I & $\nabla$ & $N$ & - & 0 & $N$ & - & $\nabla$ & - & - & 0 & 0 & 0 & $\sim$ & $\infty$ \\
\hline & $\Sigma$ & $N$ & $n$ & $n$ & $m$ & $n$ & $m$ & 6 & $N$ & $N$ & - & 0 & - & - & $\bar{\nabla}$ \\
\hline \multirow{2}{*}{$\begin{array}{l}\text { aे } \\
\text { ń }\end{array}$} & LI & $\nabla$ & $r$ & 0 & - & 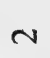 & - & - & 0 & $N$ & 0 & 0 & 0 & - & $\underline{1}$ \\
\hline & $\Sigma$ & 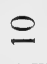 & $N$ & $n$ & - & $\nabla$ & $\infty$ & - & - & $N$ & - & $m$ & 0 & 0 & $\stackrel{\vartheta}{\nabla}$ \\
\hline \multirow{2}{*}{$\begin{array}{l}\text { à } \\
\dot{+}\end{array}$} & L & - & $m$ & $N$ & 0 & $\sim$ & $N$ & $m$ & - & - & 0 & 0 & - & 0 & $\underline{0}$ \\
\hline & $\Sigma$ & $\nabla$ & $r$ & - & - & 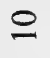 & $\nabla$ & $n$ & - & - & - & N & - & 0 & $\underset{\sim}{\infty}$ \\
\hline \multirow{2}{*}{$\begin{array}{l}\vec{a} \\
\dot{1} \\
\stackrel{a}{ }\end{array}$} & I & $m$ & 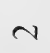 & 0 & 0 & $\nabla$ & $m$ & $n$ & $m$ & - & 0 & 0 & 0 & 0 & $\bar{v}$ \\
\hline & $\Sigma$ & 0 & $\nabla$ & $n$ & $n$ & 0 & $\nabla$ & $n$ & $m$ & - & 0 & - & 0 & - & $\bar{\nabla}$ \\
\hline \multirow{2}{*}{$\begin{array}{l}\text { a } \\
\stackrel{a}{a}\end{array}$} & LI & $m$ & $N$ & - & $m$ & 0 & - & 0 & 0 & 0 & 0 & 0 & 0 & 0 & 으 \\
\hline & $\Sigma$ & 0 & $\infty$ & $n$ & $\nabla$ & $n$ & $\infty$ & $\nabla$ & 0 & 0 & 0 & $N$ & 0 & $m$ & $\dddot{n}$ \\
\hline \multirow{2}{*}{$\frac{\hat{\alpha}}{a}$} & 도 & $m$ & 0 & 0 & - & 0 & - & $\sim$ & - & 0 & 0 & 0 & - & 0 & $a$ \\
\hline & $\Sigma$ & $m$ & $\nabla$ & $m$ & - & - & $n$ & 0 & $\nabla$ & - & 0 & - & $N$ & 0 & $\bar{m}$ \\
\hline \multirow{2}{*}{$\begin{array}{l}\bar{a} \\
\dot{\rho}\end{array}$} & II & $m$ & - & - & 0 & $m$ & 0 & $N$ & $r$ & - & 0 & 0 & - & 0 & $\underline{a}$ \\
\hline & $\Sigma$ & $m$ & 6 & 0 & - & $m$ & 6 & $\sim$ & - & $n$ & 0 & 0 & $\sim$ & 0 & શิ \\
\hline & $\frac{\frac{1}{0}}{3}$ & 仓̊ & $\frac{\Xi}{\circlearrowright}$ & $\begin{array}{l}\bar{N} \\
\text { Õ }\end{array}$ & $\begin{array}{l}\stackrel{\infty}{N} \\
\stackrel{d}{D}\end{array}$ & $\underset{\sim}{\stackrel{\Xi}{\Xi}}$ & $\underset{\Xi}{\Xi}$ & $\frac{\infty}{\frac{\infty}{5}}$ & $\begin{array}{l}\approx \\
\tilde{\Xi} \\
\approx\end{array}$ & D & $\begin{array}{l}\infty \\
0 \\
0\end{array}$ & $\frac{n}{0}$ & $\begin{array}{l}\text { ป } \\
0 \\
0 \\
1\end{array}$ & $\begin{array}{l}\text { సे } \\
0 \\
0\end{array}$ & $\begin{array}{l}\frac{w}{0} \\
\text { d } \\
3 \\
\bar{z}\end{array}$ \\
\hline
\end{tabular}




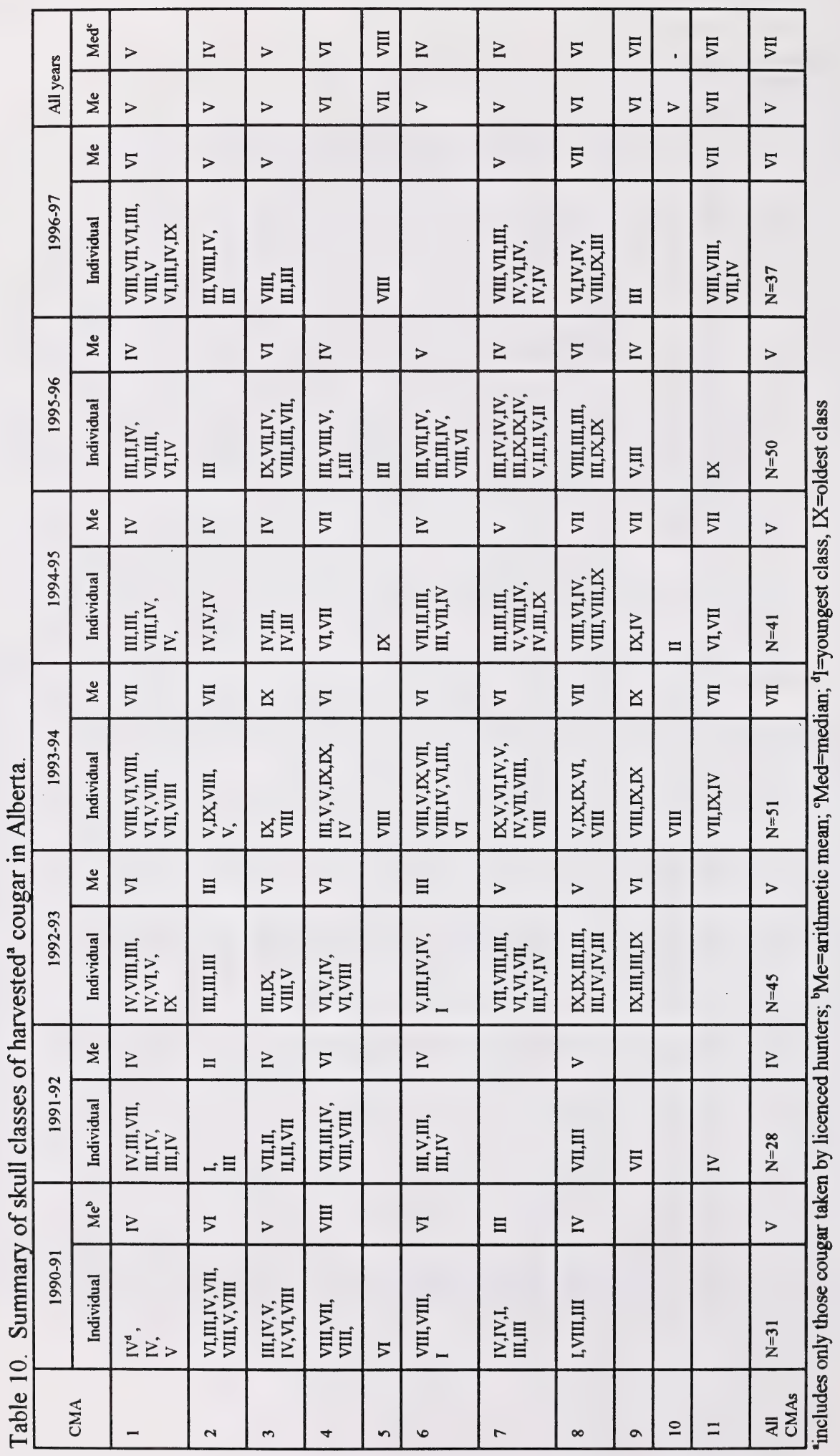


III,IV)--43 percent; 146 mature adults (classes V-IX)--52 percent.

\subsection{Total Mortalities}

Cougar deaths result from natural causes including predation by adult male cougar and accidents during attacks of prey (AFWD 1992, Ross et al. 1995), illegal and problem wildlife kills, disease, accidental captures by wolf and coyote trappers and other incidental causes. All man-caused mortalities must be registered and the resulting data during 19901997 are in Table 11. Legal hunting harvest accounted for 60 percent (1994-95) to 87 percent (1990-91) of total mortalities on a yearly basis (overall [7 yrs.]=76 percent). Harvested cougar formed the greatest proportion of reported mortalities in CMAs 3 (90 percent), 8 ( 89 percent) and 7 ( 85 percent).

\section{DISCUSSION}

Judging by comments following the 1990-91 hunt (J.R.Gunson, Alberta Fish and Wildlife Division, unpubl. rep., 1991), most aspects of the new system were appreciated by cougar hunters. In a hunter-opinion survey at the end of the hunt with its longer season and area quotas, most hunters thought season length ( 3 months) was about right and supported the area quota concept. Analysis of hunt statistics on a-before (prior to 1990-91) and-after basis, indicate a much improved situation. First, harvest of cougar north of the Bow River at Calgary, increased from 29 percent to 41 percent of the provincial total. Earlier closures in popular Cougar Management Areas in the south forced more hunters to explore and hunt in the north. Second, male cougar proportion in the harvest increased from 57 percent to 71 percent. The knowledge that shooting a female could close the season earlier (that is, female quota less than total quota) resulted in more effort to find and harvest the male. Third, hunter success increased from an average of 24 percent during 1973-1990 to 36 percent during 1990-1997. The longer season eliminated the lack of harvest related to unsatisfactory snow and temperature conditions.

Hunters have expressed dissatisfaction with the registered-letter announcement of 


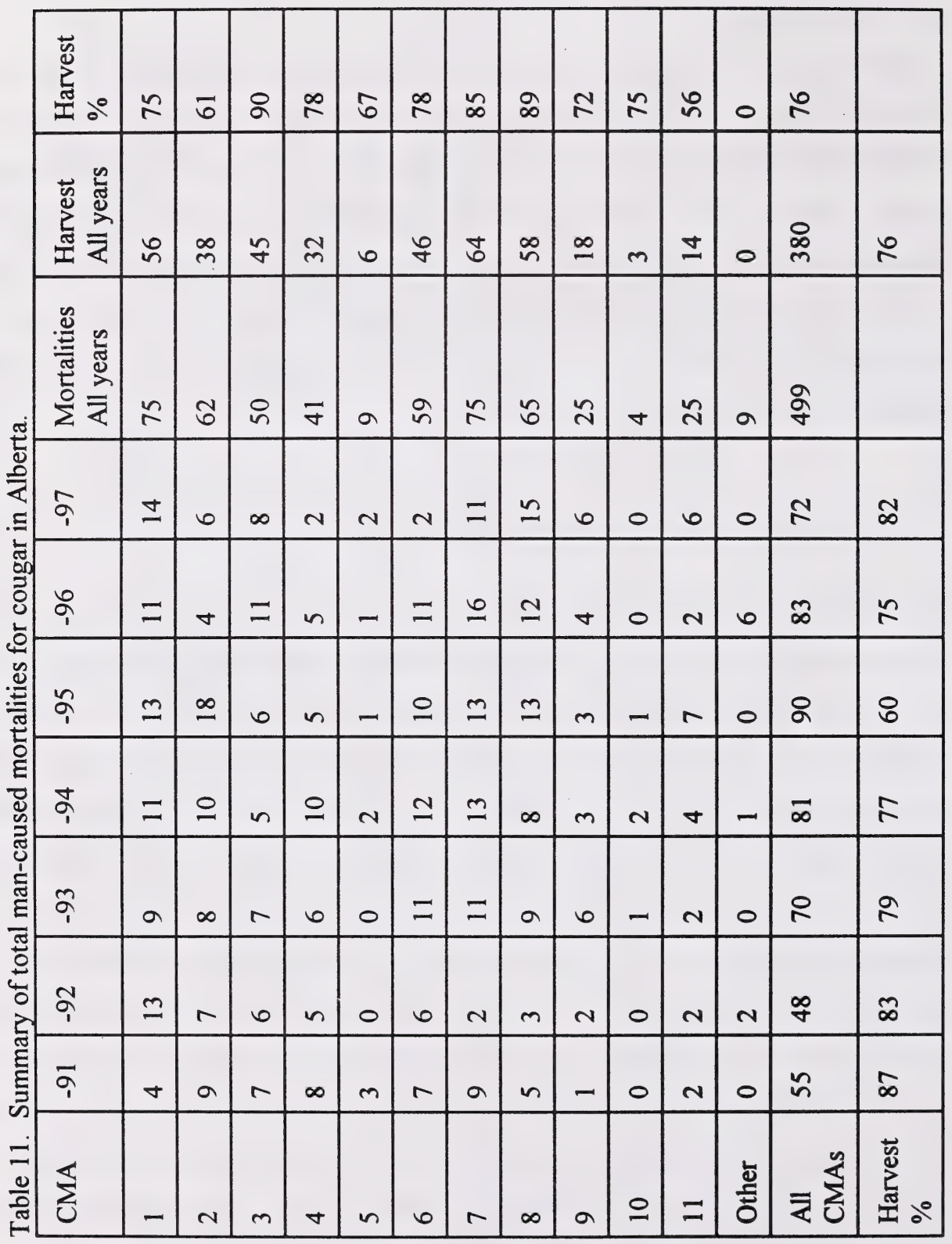


closure. The letters, mailed the day of, or the day following, discovery of quota attainment (usually 2-3 days after the quota-reaching cougar is taken), are received by licencees several days after mailing. A 5-day grace period has been allowed because of the mailing requirement. The mailings are also costly (1994-95, 7 closures as of end-January, 146 licencees, 1022 letters, cost of $\$ 4,599$.). Serious consideration should be given to placing the onus on the hunter to access the $1-800$ process to determine closure and eliminating letters.

In addition to the improvement in sex ratio of harvested cougar, the lack of decline in average skull age (Table 10) indicate the overall increase in cougar harvest provincially has not stressed regional cougar populations. The quota harvest system is effective at regulating regional harvests of cougar; and harvest rates (hunting--10 percent, man-caused mortality-- 15 percent), lack of population declines and age data suggest that population estimates were not excessive. Nevertheless, observations of fewer large adult males by experienced outfitter-guides may mean hunting in some areas is approaching the allowable limit. Monitoring of cougar harvest should be continued. 


\subsection{LITERATURE CITED}

Alberta Fish and Wildlife Division. 1992. Management plan for cougar in Alberta. Wildlife Management Planning Series. No. 5. Edmonton. 91pp.

Greer, K. 1972. Wildlife laboratory. Montana Dep. Fish and Game, Job Progress Rep. W-10-R-3, No. L-1: 15-32.

Ross, P. I. and M. G. Jalkotzy. 1992. Characteristics of a hunted population of cougars in southwestern Alberta. Journal of Wildlife Management 56: 417-426.

Ross, P. I., M. G. Jalkotzy and J. R. Gunson. 1996. The quota system of cougar harvest management in Alberta. Wildlife Society Bulletin 24: 490-494.

Ross, P. I., M. G. Jalkotzy and P-Y. Daoust. 1995. Fatal trauma sustained by cougars, Felis concolar, while attacking prey in southern Alberta. Canadian FieldNaturalist 109: 261-263. 


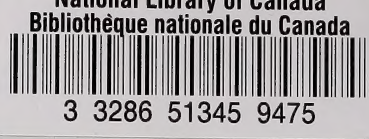

